173-196

\title{
COMUNICACIÓN INTERCULTURAL MEDIADA: CONSTRUCCIÓN DE REALIDAD A TRAVÉS DE UN ANÁLISIS CRÍTICO Y COMPLEJO DE LOS DISCURSOS PERIODÍSTICOS ENTRE CHILE Y PERÚ ${ }^{1}$
}

Mediated intercultural communication: the construction of reality through a critical and complex analysis of the journalistic discourses between Chile and Peru

\author{
Rodrigo Browne Sartori* \\ Constanza Yáñez Duamante**
}

\section{Resumen}

La acción de los medios de prensa de construir y representar realidades socioculturales genera — en reiteradas ocasiones — relaciones desiguales, promoviendo e institucionalizando unas identidades en desmedro de otras. La situación se complejiza cuando se trata de países vecinos, con sus respectivas tradiciones socio-histórico-culturales, pasados comunes y límites bisagra. Bajo este escenario se analizaron las producciones noticiosas de cobertura nacional publicadas en los periódicos de mayor tirada de dos países limítrofes: "El Mercurio" de Chile y "El Comercio" de Perú. De este modo, y por medio de una herramienta metodológica ligada al Análisis Crítico y Complejo del Discurso, esta investigación busca comprender los procesos de construcciones noticiosas y representaciones que los medios de prensa chilenos y peruanos hacen en torno a los “discursos de la diferencia” que se institucionalizan en la relación entre ambas naciones.

Palabras clave: Comunicación intercultural mediada, construcción social de la realidad, contexto binacional y análisis crítico y complejo del discurso.

Abstract

The action of the press media of constructing and representing socio-cultural realities generates —on numerous occasions - unequal relationships, which promote and institutionalize certain identities in detriment of others. The situation becomes more complex when it deals with neighboring countries and their corresponding socio-historical-cultural traditions, shared pasts, and "hinge-like" borders. Under this scenario, the production of national news by two nationwide press media of the neighboring countries will be analyzed: "El Mercurio" of Chile and "El Comercio" of Perú. In this manner, and using a methodological tool based on the Critical and Complex Analysis of the Discourse, the main objective of this research is to understand processes of construction of news and representations that the Chilean and Peruvian press make linked to the "discourse of the differences” which have been institutionalized in the relationship between them both.

Key words: Mediated intercultural communication, social construction of reality, binational context and critical and complex analysis of the discourse.

Una bisagra permite que una puerta se abra o se cierre como las fronteras que suelen separar pero también unir (Acosta, 2010). Bajo esta relación es posible

\footnotetext{
${ }^{1}$ Esta investigación forma parte de la ejecución del Proyecto Fondecyt № 11070264. Contó además con el apoyo de la Comisión Nacional de Investigación Científica y Tecnológica de Chile (CONICYT).
} 
comprender la comunicación intercultural mediada, como puertas y ventanas que se abren y cierran a los discursos mediáticos, construyendo realidades sociales e identidades conjuntas y fraccionadas, cercanas y distantes, propias y ajenas.

Alain Touraine (1997) precisa que a fines del siglo XIX, durante la revolución industrial y en un contexto de modernización económica, la nación que hasta aquel momento concertaba la voluntad general y el pluralismo de opiniones se redujo a una unidad nacional y a su afirmación: el nacionalismo. La conciencia de lo nacional, sumado a los intereses económicos, ha irrumpido en los medios de comunicación, amenazando la complementariedad integracionista en pos del segregacionismo. Aquello impulsado mayoritariamente por grupos dominantes legitimados, con acceso al discurso público y fuerte poder de influencia. Este rol es interpretado con precisión por los medios de comunicación, quienes además — como empresas productoras de información — se encuentran inmersos en el sistema capitalista, formando parte y siendo promotores del mismo, reproduciendo como valores periodísticos aquellos valores económicos e ideológicos de su interés.

Las estrategias de dominación discursivo-ideológica apuntan a controlar estructuras mentales, de ahí que el poder que ejercen los medios resulte ser simbólico y persuasivo. "Creer que las relaciones sociales y comunicativas no son un lugar de negociación donde los grupos dominantes y los emergentes entran en conflicto es una ilusión. Es importante desenmascarar quién se atribuye el poder de definirnos” (Rodrigo, 1999:55).

Desenmascarar el poder y sus estrategias de dominación presentes en los medios, es tarea de todos quienes de algún modo formamos parte del entramado comunicación. Sobre estas relaciones, se exponen aquí parte de los resultados de la investigación aplicada en 2010, denominada "Comunicación intercultural mediada de carácter binacional: construcción de la noticia a través de un análisis crítico y complejo del discurso en la prensa diaria de cobertura nacional de Chile y Perú”. En ésta se abordan los procesos de construcción noticiosa y las representaciones que hacen los medios de prensa sobre la dualidad Nosotros/Ellos, en este caso entre los países limítrofes Chile y Perú en relación a los discursos de la diferencia que se institucionalizan en torno al "otro".

Tras la investigación se puede aseverar que, luego de centenas de años, pocos parecen recordar o saber que peruanos y chilenos —en otro tiempo- lucharon juntos por objetivos comunes y fundamentales para la conformación de sus respectivas repúblicas. En contra, la memoria colectiva nacional —organizada y promovida desde los estados - se sobrealimenta de uno de los conflictos más desastrosos de las relaciones entre ambos países: la Guerra del Pacífico ocurrida entre 1879 y 1883, también conocida como la Guerra del Guano y del Salitre. Una guerra que enarboló campañas terrestres y marítimas, batallas, héroes y villanos, valientes y cobardes. Como resultado, hoy Chile limita al norte con Perú y Bolivia, mientras que antes de la guerra sólo limitaba con Bolivia. Pero más allá de los límites, más allá de botines 
de guerra y de héroes muertos en combate, este resultó ser un conflicto bélico cruento tanto para Chile como para Perú, acarreando consigo la depresión de una postguerra y un sinfín de distorsiones transmitidas por los canales oficiales de la educación escolar y universitaria; nomenclatura de calles, plazas y avenidas; fiestas cívicas; museos y monumentos; manuales y libros de historia que se enlazan en íntima unidad con el interés nacional que se persigue, manteniendo al mismo tiempo un compromiso con los hechos de un pasado reconstruido "objetivamente" por historiadores académicos (Contreras, 2006: en línea). Hoy este hecho continúa inclinando la balanza, reproduciendo y configurando — también a través de los medios - gran parte de las identidades nacionales de Chile y Perú, olvidando aquellos acontecimientos de conciliación como si no existiesen a lo largo de estas historias mutuas y paralelas.

Lo anterior es corroborado en el análisis de las noticias publicadas en los diarios El Mercurio y El Comercio, pertenecientes a grandes conglomerados de la industria comunicacional económica de Chile y Perú respectivamente. De estos medios se seleccionaron las construcciones noticiosas referidas de forma común a ambos países, y entre ellas las de mayor recurrencia y permanencia durante el periodo de estudio, resultando como temática más abordada el diferendo marítimo que sostienen Chile y Perú ante la Corte Internacional de Justicia de La Haya desde inicios de 2008.

Los medios analizados fortalecen las respectivas identidades de sus EstadoNación, a través de la construcción de acontecimientos noticiosos concernientes a Chile y Perú, basados mayoritariamente en situaciones que invitan a la segregación. Del análisis se desprende que las construcciones despliegan diversas estrategias discursivas, principalmente implícitas, y vinculadas a destacar aquellas características o situaciones positivas sobre el Estado-Nación propio, en desmedro del ajeno. En este sentido es pertinente recordar las palabras de Teun van Dijk en relación a que los medios de comunicación son la institución principal de (re)producción ideológica, "probablemente más importantes que el sistema educativo propiamente dicho aunque a decir verdad hemos visto que los medios de comunicación y, por lo tanto, los periodistas, no realizan esta tarea en solitario” (Van Dijk, 1997:53).

\section{AlgunAS IDEAS BASES SOBRE COMUNICACIÓN Y CULTURA}

Resulta innegable la idea de comprender a la comunicación como el motor fundamental de las relaciones humanas, capaz de producirlas y reproducirlas. Sin embargo, esta idea puede parecer de sumo general. Definir la comunicación es un asunto complejo sobre el que acotadas investigaciones han puesto todos sus esfuerzos, sin llegar a convenir respecto al anhelado acuerdo conceptual. Vilém Flusser (2003) expone una mirada crítica al intentar responder ¿Qué es la comunicación? Para el autor, la comunicación es un proceso artificial generado a partir de invenciones e instrumentos erigidos en símbolos que, a su vez, han sido ordenados en códigos. La comunicación humana es una suerte de artificio del hombre frente a la soledad dispuesta hacia una muerte inminente. 


\section{Rodrigo Browne y Constanza Yáñez}

Similar situación se observa en relación a la cultura, que también como construcción del ser humano, ofrece mecanismos de resistencia ante su conceptualización. Clifford Geertz (1973), expone que la cultura es una urdimbre que el mismo hombre ha tejido y que su análisis vendría a ser una ciencia interpretativa en busca de significaciones y no de leyes. Para García Canclini, la cultura comprende un conjunto de procesos sociales de producción, circulación y consumo de la significación en la vida social; mediante los cuales dos o más grupos representan o intuyen lo social, concibiendo y gestionando relaciones diferentes con otros.

La cultura no es apenas un conjunto de obras de arte, ni de libros, ni tampoco una suma de objetos materiales cargados con signos y símbolos. La cultura se presenta como procesos sociales, y parte de la dificultad de hablar de ella deriva de que se produce, circula y se consume en la historia social. No es algo que aparezca siempre de la misma manera (García Canclini, 2004:34).

En este conjunto de procesos sociales se crean las interrelaciones culturales, las diferencias y puntos de orden de las dispersiones de los grupos sociales, o como diría Bourdieu (1990), el espacio de la reproducción social y organización de las diferencias. Siguiendo con la idea anterior, es oportuno destacar la postura interaccionista que ofrece Rodrigo Alsina, para quien la cultura debe su existencia y permanencia a la comunicación, considerando que es en la interacción comunicativa entre las personas donde ésta se manifiesta (Rodrigo, 1999:67).

Podemos entonces percibir el complejo entramado que se construye en relación a la cultura. Y dado que "el hombre construye una realidad y se permite definir (y no explicar) lo que él entendió y quiso entender como cultura” (Browne, 2006:228), podemos decir que la cultura se presenta indefinida, imprecisa e indeterminada por esencia. Escapando a todo plan de definición universal, la cultura invita a ser analizada desde realidades diversas; invita a quebrantar tradiciones institucionales y etnocéntricas construidas desde la diferencia, desde “otros”.

\section{CONSTRUCTORES DE REALIDAD SOCIAL}

Con la idea de releer aquellas conceptualizaciones — cultura y comunicación por ejemplo - que entendemos como procesos construidos socialmente, y en relación a los objetivos que motivan esta investigación, destacamos las nociones expuestas por Berger y Luckmann (1972), quienes plantean que el hombre tiene una relación dialéctica con la realidad, de la que es producto y productor. El constructivismo sostiene que no existe una sola realidad, sino realidades conformadas a raíz de la suma de contextos y procesos. Sobre esta relación Berger y Luckmann plantean su teoría de la institucionalización de la realidad social, basada en procedimientos operativos secuenciales tendientes a la habituación del hombre. La institucionalización, por su sola existencia, ejerce control en el comportamiento humano, estableciendo pautas definidas con antelación y canalizadas en una dirección determinada, en contradicción a muchas otras que podrían darse. 
No debería sorprendernos, pues, que exista una profunda afinidad entre aquellos a quienes les interesa retener las posiciones de poder establecidas y los elencos que administran las tradiciones monopolizadoras para el mantenimiento de los universos. En otras palabras, las fuerzas políticas conservadoras tienden a apoyar los reclamos monopolizadores de los expertos universales, cuyas organizaciones también monopolizadoras tienden a ser políticamente conservadoras (Berger y Luckmann, 1972:156).

La construcción social de la realidad trae consigo ideologías dominantes como armas para intereses sociales y económicos, interviniendo en toda la esfera sociocultural e inevitablemente, en las relaciones comunicativas. Los medios — desde un sitial privilegiado - fabrican realidades fragmentadas y definen imágenes que se instauran e institucionalizan como realidades para una sociedad, realizando interpretaciones mayoritariamente hegemónicas. Para entender esta problemática es fundamental comprender a la institución comunicativa mediante los procesos y contextos que han originado su conformación como constructores de representaciones sociales.

\subsection{COMUNICACIÓN INTERCULTURAL Y MEDIACIÓN SOCIAL}

Según Miquel Rodrigo Alsina, "las formas de vida actuales son propias de una sociedad que, para funcionar, depende cada vez más de la mediación social que se encomienda a las instituciones comunicativas" (1989:47). Las noticias que se construyen y emiten socialmente por los medios, sólo reflejan un puñado de acontecimientos, o sea, de hechos que rompen con lo ordinario dentro de una "normalidad". Los criterios que deciden qué merece ser noticia y qué no, corresponden de manera preferente al poder institucional de los medios de comunicación. En consecuencia el izamiento u omisión de una noticia puede deberse, más a los intereses de la empresa comunicativa a la que se adscribe el periodista, que a los propios intereses del profesional. Rodrigo Alsina, basado en sus estudios, señala que "se ha demostrado que los comunicadores adaptan sus puntos de vista a la exigencia de la organización y esto explica el tipo de contenido producido por un medio" (1989:189-190).

A ello se agrega la diferencia sociocultural que se presenta como un factor trascendente para las actuales interrelaciones comunicativas, dada su intensificación gracias a la globalización mediática, al desarrollo tecnológico y al incremento de ciudadanías multiculturales. Si bien es cierto nos hemos conectado, también se ha dado pie a posturas universalistas, excluyentes y tremendamente etnocéntricas; las que a su vez, han abierto puertas y ventanas de prejuicios y estereotipos reproducidos por los distintos discursos mediáticos. La comunicación intercultural, comprendida como la que surge entre pueblos con diferentes sistemas socioculturales, sugiere que no se debería apelar a la construcción de estructuras o categorías políticas, sino al aprendizaje, a la reflexión y el análisis. La comunicación intercultural no es un ejercicio de simplificación, ya que frente a sociedades culturalmente distintas, hay también 


\section{Rodrigo Browne y Constanza Yáñez}

códigos distintos que implican una relación dialéctica entre un nos-otros y unos otros. La negativización y distorsiones que los medios construyen en relación a las diferencias ocurren generalmente por desconocimiento del otro o por factores situacionales nacionales, económicos, históricos-conflictivos, políticos, educacionales, etc. En el caso de los discursos mediáticos entre Chile y Perú, todas las razones mencionadas parecen ser parte en mayor o menor medida de las distorsiones blandidas a través de los poderes dominantes de la empresa comunicativa. Estrella Israel Garzón señala que la situación se agrava en periodos de crisis o de conflicto, "cuando un prejuicio o un estereotipo como simplificación racial, puede desembocar en dicotomías trágicas, de la que la historia está plagada de ejemplos” (2006:42).

3. NOCIONES DEL CONTEXTO Y ESTATUS QUO DE LA CONFLICTIVIDAD ENTRE CHILENOS Y PERUANOS

Los medios de comunicación, como actores y constructores sociales de realidad, se ven expuestos a diversos escenarios socio-contextuales en relación a los discursos de la diferencia chileno-peruano. Como mencionábamos anteriormente y desprendido del análisis realizado, la conflictividad entre Chile y Perú parece mantenerse a nivel contextual discursivo, apoyado por la vieja herida de la Guerra del Pacífico. La Historia del Perú Contemporáneo (2007), de los autores Contreras y Cueto, resulta ser una de las escasas versiones con tintes cosmopolitas que circulan sobre este hecho. En relación al inicio del conflicto bélico se señala

Desatado el conflicto, con la ocupación chilena del litoral boliviano, Perú se puso del lado de Bolivia: fuera en cumplimiento del Tratado de 1873, según la historiografía peruana; o porque también ambicionaba el salitre del territorio boliviano, a fin de reconstruir su ansiado monopolio de los fertilizantes, según la chilena. He aquí a tres gobiernos contemplando el salitre como miraría un hambriento viajero un trozo de carne tierna (163).

En el contexto del análisis de esta investigación, se pudo evidenciar que se insiste - por medio de diversos canales oficiales - en encapsular en la memoria colectiva aquellos hechos más negativos de las relaciones entre Chile y Perú, siendo esto reflejado y reproducido por los medios.

El historiador peruano Joseph Dager (“Enemigos íntimos”, El Mercurio Online,) afirma que los nacionalismos chileno-peruano se afianzan innegablemente a partir del resultado de la Guerra del Pacífico; su connacional Carlos Contreras agrega que la Guerra del 79', sus secuelas y su memoria, marcaron la interrelación entre ambos países, "creando recelos, armamentismos y disminuyendo el comercio que podría haber existido” (Entrevista personal, 2010²). Sobre estas relaciones, nos preguntamos por qué ciertas memorias oficiales nacionalistas permanecen en la

\footnotetext{
2 Entrevista personal, realizada por Constanza Yánez Duamante, al Dr. Carlos Contreras, profesor de Historia Económica de la Pontificia Universidad Católica del Perú (20-12-2010). Todas las entrevistas personales de este artículo fueron realizadas por la misma investigadora.
} 
quietud del estatus quo; por qué otros países como Francia y Alemania con conflictos históricos de grandes proporciones conviven relativamente en armonía, mientras Chile y Perú parecen tener fuertemente afectada la confianza y el respeto por el otro.

Si acudimos a los sistemas educativos chileno y peruano el escenario es complejo, pues vemos que las narraciones de la historia y los discursos en la enseñanza de la misma están repletos de distorsiones. Carlos Contreras comenta que el proceso hacia una lectura más realista y responsable de la guerra es aún lento y que los relatos que se hacen en el Perú son sobre todo militares. En este sentido la educación, como constructora de individuos que más tarde conformarán sus propios discursos y ocuparán determinados roles en las sociedades a las que se adscriban, se presenta como una de las estructuras más preocupantes del asentamiento del chovinismo.

Podría decirse que a todos los niños debe contárseles la misma historia. Se sigue que el orden institucional en expansión elabora una cubierta correlativa de legitimaciones, extendiendo sobre ella una capa protectora de interpretación tanto cognoscitiva como normativa. Estas legitimaciones son aprendidas por las nuevas generaciones durante el mismo proceso que las socializa dentro del orden institucional (Berger y Luckmann, 1972:85).

El peso que tiene el sistema educativo en el modelo cultural dominante caracterizado como blanco, adulto, masculino y de clase media- suele presentarse en todos los libros de textos y materiales complementarios escolares. Se vuelve evidente la necesidad de generar conciencia sobre el estado actual e implicancias sociales de los errores en la historiografía, puesto que han permitido profundizar las diferencias entre países limítrofes.

A las oligarquías peruana y chilena, este estado de cosas les es conveniente. Al hacer mención de la guerra en los medios, apoyados por la historia y la educación, se configura un servicio a los detentadores del poder, quienes al blandir estos argumentos mantienen el status quo (Cortez, entrevista personal: 2010). ${ }^{3}$

Fernando Ramírez es uno de los autores del libro de sexto año básico del Ministerio de Educación de Chile, Historia, Geografía y Ciencias Sociales. En este texto, como en gran parte de los manuales escolares de este país, se explica que la Guerra del Pacífico se inició porque las autoridades bolivianas no respetaron el Tratado de 1874. Sin embargo, el único párrafo que se dedica a la guerra se inicia así: "En este periodo Chile tuvo complejas relaciones internacionales” (Ramírez y Silva, 2019:182). El autor explica que es una frase que costó insertar y que discrepa de dicha organización curricular:

\footnotetext{
${ }^{3}$ Dr. en Historia y Director del Museo Regional de Atacama, Chile (24-11-2010).
} 


\section{Rodrigo Browne y Constanza Yáñez}

El proceso educativo chileno de la historia no apunta a las interrelaciones o integración entre países, sino que tiene como gran objetivo la obtención de resultados para sistemas de medición, como la $\mathrm{PSU}^{4}$ o el SIMCE ${ }^{5}$. La torpeza del Ministro Lavín ${ }^{6}$ lo demuestra, con eso de ponerles banderitas de colores a los colegios para medir sus rendimientos en dichos sistemas. Hoy en día tiene mucha más influencia lo que hacen los medios que lo que pueda llegar a hacer un texto escolar (Entrevista personal: 2010). ${ }^{7}$

En la actual época de globalización el rol educativo parece ser preferentemente responsabilidad de los medios de comunicación, quienes sustentan el chovinismo como funcionalidad política, copando al mismo tiempo las estructuras sociales. El historiador Guillermo Cortez afirma que conocernos de mejor manera mediante una historia crítica posibilitaría que el sistema educacional fuera más integrador, equiparando las similitudes y potencialidades con las diferencias. "De esta forma y con el concurso de los medios de comunicación, podríamos pasar a una etapa de comprensión y desarrollo cooperativo entre países limítrofes. Herramienta verdadera de desarrollo integral de los pueblos” (Entrevista personal, 2010). Por su parte, Fernando Ramírez centra las posibilidades de una futura distensión en los mundos académicos, desde donde cree posible cambiar los discursos más belicistas que hasta hoy perduran.

Es innegable que los discursos mediáticos construyen versiones del mundo, de ahí la importancia de la comunicación intercultural mediada de carácter internacional para el desarrollo integracionista de las naciones. Tornándose indispensable que, quienes integramos labores sociodiscursivas, intentemos acabar con las hipertrofias al comunicar la diferencia y adoptemos posturas interaccionistas, plurales y de respeto mutuo. En este sentido, el análisis crítico de los discursos se plantea como una herramienta capaz de descubrir y revelar ciertas estrategias de los medios de comunicación en relación a la alteridad, puesto que muchas de las distorsiones que se reproducen a través de los discursos periodísticos son presentadas de modo sutil e implícito.

\section{Propuesta METOdOLÓGICA: ACD y ACCD}

Para intentar comprender ciertos procesos de representación de realidad mediante construcciones noticiosas binacionales, a través de los discursos mediáticos de mayor lectoría en Perú (El Comercio) y Chile (El Mercurio), se utilizó una metodología de investigación de carácter cualitativa hermenéutica. La propuesta metodológica se planteó en dos etapas, en las que se intentó aplicar y

\footnotetext{
${ }^{4}$ Prueba de Selección Universitaria.

${ }^{5}$ Sistema de Medición de Calidad de la Educación.

${ }^{6}$ Joaquín Lavín, ex Ministro de Educación de Chile desde inicio del Gobierno de Sebastián Piñera en 2010.

${ }^{7}$ Historiador y docente de la Universidad de Chile (22-12-2010).
} 
presentar un análisis crítico efectivo, capaz de sugerir soluciones y alternativas ante un imperio discursivo etnocéntrico nacionalista y ante sus diversas estrategias de manipulación, en el escenario del tratamiento noticioso de la prensa peruana y chilena. A continuación pasamos a explicar ambas etapas metodológicas.

\subsection{SOBRE EL ACD}

El análisis crítico del discurso (ACD) es un tipo de investigación analíticodiscursiva dedicada a estudiar el abuso de poder, el dominio y la desigualdad social, sus formas de producción y reproducción mediante el texto y el habla desde contextos sociopolíticos-culturales. Tomando para ello una postura crítica oposicionista y de resistencia.

Teun van Dijk, quien ha desarrollado ampliamente el ACD, ha dedicado sus más recientes estudios al ACD en los medios, centrándose en las noticias y la prensa. Van Dijk sugiere, que los medios en el contexto sociopolítico, se han situado al lado de los poderes. Según el autor, "quienes controlan el discurso público controlan ampliamente la mentalidad social, e indirectamente la acción pública; y, por consiguiente, controlan también la estructura social” (2009:175). Para conocer estas estrategias de dominación discursiva es necesario un análisis en detalle, tanto del texto como del contexto, puesto que casi todo afecta nuestras opiniones y actitudes. "Un análisis tan complejo del discurso no se limita al análisis textual, sino que tiene también en cuenta las relaciones entre las estructuras de texto y habla por una parte y, por otra, su 'contexto' cognitivo, social, cultural o histórico" (1997:30). Para objeto de la investigación que se expresa en este artículo, se contempló la aplicación de una matriz basada en el ACD y considerada, principalmente, en y de los trabajos de van Dijk.

CuAdro 1. Planos Y NIVELES DEL ACD EMPLEAdO:

\begin{tabular}{|l|l|}
\hline Plano Significado/texto. Nivel temático \\
\hline $\begin{array}{l}\text { Nivel de Significados } \\
\text { Globales. }\end{array}$ & \\
\hline \multirow{2}{*}{$\begin{array}{l}\text { Nivel de } \\
\text { Significados } \\
\text { Locales. }\end{array}$} & De carácter implícito o indirecto: \\
\cline { 2 - 2 } & \\
\cline { 2 - 2 } Plano Formal/texto-contexto. & De carácter explícito o directo: \\
\hline Estructuras Formales Sutiles. & \\
\hline $\begin{array}{l}\text { Nivel } \\
\text { Contextual. }\end{array}$ & \\
\hline
\end{tabular}

Se proyectan - para posteriormente corroborarse y validarse en los siguientes pasos de la matriz — los tópicos de texto más recurrentes, los significados explícitos e implícitos; así como las estructuras formales sutiles y las representaciones mentales 
de la memoria a largo plazo. Se aborda un conjunto de pasos respetando las categorías específicas de análisis, que se aplica desde lo general a lo particular en aspectos formales y de significados y se dividen, a su vez, en cuatro niveles presentados en dos planos (Cuadro 1).

\subsubsection{NIVEL TEMÁTICO DE SIGNIFICADOS GLOBALES}

En este plano, busca conocer cuáles son los temas a los que se les atribuye importancia en la noticia. Se presenta una especie de resumen que incluye de qué trata y cómo se emite la construcción noticiosa. Generalmente no se reconoce con tanta evidencia, por lo que debe ser inferido del propio discurso. De este primer nivel temático se desprenden, implícitamente, las variables temáticas, también denominadas tópicos de texto por van Dijk, las que se dilucidarán a posteriori en la segunda etapa. Para este autor los tópicos o variables son importantes porque entregan una coherencia global al discurso y, además, es la información que es recordada de mejor manera, organizando la forma en que se representan los acontecimientos en las memorias personales de las experiencias del día a día y su correlación tiempo/espacio.

Nivel de Significados Locales. Aquí se plantea analizar los discursos periodísticos mediante el significado concreto de las palabras. Es posible encontrar cómo mediante construcciones semánticas se destacan las "buenas cosas" y se olvidan "las malas" y a la inversa, en relación a la diferencia cultural de -en este caso- un país limítrofe. Este nivel de significados locales se divide en dos categorías: de carácter implícito y explícito.

-De carácter implícito: no se expresa la "diferencia” de manera evidente y es necesario inferirla relacionando los significados de las palabras del texto, ya que se presupone que los imaginarios están construidos.

-De carácter explícito o directo: se presentan informaciones evidentes, que se encuentran expresadas con claridad en las palabras del texto; lo cual permite observar las elecciones estilísticas, que suelen acarrear implicaciones ideológicas dando a entender la opinión del periodista y/o del medio de comunicación en general.

\subsubsection{PLANO FORMAL/TEXTO-CONTEXTO}

Estructuras formales sutiles: se investiga cómo operan los aparatos ideológicos formales del discurso que pueden emitir falacias u omitir información crucial, construyendo modelos sesgados e interesados de acuerdo a los discursos de dominación y sus fuentes. En general se refiere a la construcción diaria de las realidades y cómo son percibidas. Hablamos de aquellas sutilezas de carácter indirecto a raíz de la semántica utilizada.

Nivel contextual: aquí se investigan las representaciones mentales de la memoria a largo plazo, sobre las que se apoya el conocimiento de la realidad, que descansan en cuestiones históricas, políticas, económicas y sociales que confirman el vínculo entre el discurso y la cultura. 


\subsection{SOBRE EL ACCD}

El carácter complejo del ACD, radica principalmente en que los discursos sociopolíticos son altamente complicados, por lo que la matriz de análisis debe ajustarse y recoger dicha complejidad. En este sentido, la aplicación de esta matriz comprende abordar no sólo elementos textuales, sino también aquellos elementos contextuales que provienen de determinados discursos públicos. El contexto tiene que ver con aquellas estructuras mentalmente representadas sobre lo social, que inciden en la producción y/o comprensión del discurso. Lo que se podría categorizar según la definición global de la situación, su espacio y tiempo, las acciones en curso, otros discursos relacionados, los actores involucrados y sus representaciones mentales: objetivos, conocimientos, opiniones, actitudes e ideologías (van Dijk, 2009). Los elementos y situaciones del entorno que se relacionan con los distintos discursos no pueden ser obviados; por ello, el análisis contextual "permite romper un sistema de relaciones discursivas dominantes, para incorporar a los discursos emergentes que permanecen especialmente en la complejidad que se da en los márgenes de la pura textualidad” (Del Valle y otros, 2008:144).

Los intertextos de los diferentes discursos otorgan sentido, pues en ellos existen complejas vinculaciones, sobre todo en el ámbito sociopolítico. Por ello, a la hora de realizar un análisis discursivo, no debiésemos prescindir del carácter complejo que lo circunscribe. El Análisis Crítico y Complejo del Discurso (ACCD) pretende entonces, comparar, aproximar, cotejar, descubrir, distanciar, confrontar o divulgar aquellas relaciones y nexos explícitos e implícitos en los discursos (Mayorga y León, 2007).

Para clarificar esta segunda etapa, se aplicará un ejercicio comparativo que se especifica desde un ACCD, logrando cotejar los mismos acontecimientos noticiosos publicados por El Mercurio de Chile y El Comercio de Perú. Esta propuesta se desprende de forma directa de los postulados expuestos sobre el ACD de Teun van Dijk en varias de sus obras y aplicaciones. Esta iniciativa también se vincula a los trabajos realizados por Carlos Del Valle a partir de la aplicación del ACD en noticias y sentencias jurídicas, compuesta por perspectivas desarrolladas por Potter y Rodríguez Villasante, tomando como eje la noción de matrices complejas que permite vincular los planos y sus niveles con la formulación de variables temáticas que rescatan los elementos recurrentes de las publicaciones en cuestión. Encuentro que se evidencia en el ejercicio comparativo que cruza los resultados de la aplicación de la matriz de ACD ( $1^{\text {a }}$ etapa) de El Mercurio y El Comercio sobre un mismo acontecimiento noticioso publicado de y entre los países en análisis. En el particular, las informaciones referidas al tema seleccionado, para construir variables temáticas comparativas que sean aplicables a las noticias de ambos países, y así identificar y obtener indicadores sobre flujos y comportamientos pertinentes para los propósitos de la investigación. 
Las variables temáticas identificadas en la investigación que centra este artículo fueron adscritas a las categorías de iguales, diferentes (“otra”), opuestas o complementarias de entre las mismas publicaciones comparadas en ambos medios. Acotamos que las categorizaciones empleadas se seleccionaron en base a su operatividad en relaciones primarias de comparación discursiva, y que fuera de ellas otras relaciones o categorizaciones que pudiesen ser relevantes para diferentes propósitos investigativos. Recordamos también que cuando se habla sobre análisis comparativo como matriz compleja, se plantea que la función de este instrumento consiste en comparar — para este trabajo- dos casos con el fin de poner de manifiesto sus disparidades y conformidades recíprocas.

-Variables iguales. Se identificaron como iguales aquellas que, en las publicaciones sobre un mismo hecho noticioso, sean utilizadas en igual o similar sentido discursivo tanto en el diario El Comercio de Perú como en El Mercurio de Chile.

-Variables diferentes. Se adscriben a esta categoría las variables discursivas que siendo identificadas comparativamente en las mismas publicaciones, sólo se manifiesten en uno u otro medio; aquellas que no posean correlación entre las dos publicaciones comparadas.

-Variables opuestas. Se refieren a variables que impliquen posturas contrarias en los discursos mediáticos entre las mismas publicaciones comparadas en los medios de estudio.

-Variables complementarias. Hablamos de elementos o componentes íntegramente relacionados e involucrados. Por ende, aquí se distinguen aquellas variables que estén relacionadas complementariamente entre sí, dentro de las mismas publicaciones comparadas en ambos medios. Estas variables podrán, sin duda, estar además adscritas a las categorías anteriores.

CUADRO 2. EJEMPLO PLANO FORMAL/TEXTO-CONTEXTO:

\begin{tabular}{|l|c|c|l|c|c|}
\hline \multicolumn{2}{|c|}{ CHILE } & \multicolumn{3}{l|}{ Título: } \\
\hline Título: & \multicolumn{2}{l|}{ Código de la noticia: } \\
\hline Código de la noticia: & Complementario & Igual & Complementario & Contrario & $\begin{array}{c}\text { Difer } \\
\text { ente }\end{array}$ \\
\hline \multicolumn{2}{|c|}{ Identificación y comportamiento de variables temáticas de un mismo acontecimiento noticioso } \\
\hline \multicolumn{6}{|c|}{ Nivel Contextual } \\
\hline Diferente & Contrario & $\begin{array}{c}\text { Guerra } \\
\text { del } \\
\text { Pacífico }\end{array}$ & & & \\
\hline & & & & & \\
\hline
\end{tabular}

\subsubsection{CRITERIOS DE SELECCIÓN}

Como ya se precisó, se analizaron dos medios de prensa escrita pertenecientes a dos conglomerados comunicacionales de gran tiraje en Chile y Perú, ambos resultan ser los periódicos de distribución nacional más antiguos de cada país: el diario chileno El Mercurio Sociedad Anónima Periodística y el diario peruano El 
Comercio Empresa Editora S.A. De las noticias, fueron seleccionadas las atinentes al diferendo marítimo que sostienen Chile y Perú ante La Haya, por tratarse de la temática más recurrente y la que se mantuvo durante el periodo de investigación: septiembre, octubre y noviembre de 2010. Las secciones de donde se analizaron las noticias corresponden principalmente a Política, Opinión, Mundo y Nacional. Se privilegiaron las informaciones de género informativo por el supuesto ajuste formal de sus construcciones y por presentar un lenguaje definido como “culto". También se seleccionaron noticias del género opinión, con la intención de obtener información sobre la valoración del medio en torno al tema investigado y conocer la correlación de dichos discursos con aquellos menos explícitos del género informativo.

\section{Resultados. Análisis de El Comercio de Perú y El MerCuRio de Chile}

De la aplicación de ambas etapas metodológicas se realizó el correspondiente análisis del material periodístico de cara a los propósitos de la investigación. Sobre esta base señalamos que el tema de mayor periodicidad y que cohesionó este análisis, la demanda de límites marítimos realizada por Perú sobre Chile ante los Tribunales de La Haya, es tratado en similar proporción de publicaciones en ambos medios. Para la primera etapa metodológica, en El Mercurio se analizaron 33 publicaciones noticiosas, mientras que en El Comercio fueron analizadas un total de 36. De estas noticias se encontraron 15 equivalencias en relación al mismo hecho noticioso publicado, éstas fueron abordadas de forma comparativa en la segunda etapa de la metodología, presentando comportamientos e identificaciones de variables temáticas en virtud a las representaciones sociales que construyen dichos medios.

CUADRO 3. PUBliCACIONES ANALIZADAS:

\begin{tabular}{|c|c|}
\hline El Mercurio - Chile & El Comercio - Perú \\
\hline \multicolumn{2}{|c|}{ Etapa metodológica 1} \\
\hline 33 Publicaciones noticiosas analizadas & 36 Publicaciones noticiosas analizadas \\
\hline \multicolumn{2}{|c|}{ Total: 69 publicaciones noticiosas } \\
\hline \multicolumn{2}{|c|}{ Etapa metodológica 2} \\
\hline 15 Equivalencias nc & osas de ambos medios \\
\hline
\end{tabular}

CUADRO 4. SECCIONES MÁS RECURRIDAS DE LAS PUBLICACIONES ANALIZADAS:

\begin{tabular}{|c|l|}
\hline El Mercurio - Chile & El Comercio - Perú \\
\hline \multicolumn{2}{|c|}{ Política } \\
\hline $55 \%$ Publicaciones noticiosas analizadas & $72 \%$ Publicaciones noticiosas analizadas \\
\hline \multicolumn{2}{|c|}{ Opinión } \\
\hline $15 \%$ & $14 \%$ \\
\hline
\end{tabular}

Acotamos que de la totalidad de publicaciones analizadas, la mayoría se encuentran adscritas a la sección Política: en El Mercurio hablamos de un 55\%, mientras en $\mathrm{El}$ Comercio de un $72 \%$. La segunda sección con más publicaciones 
sobre el criterio temático aplicado (diferendo marítimo), es Opinión: El Mercurio con un $15 \%$ y El Comercio con $14 \%$. Cabe destacar que, del periodo de estudio y a modo de ejemplo, sólo se presentará una noticia de El Comercio y una de El Mercurio con su análisis correspondiente, agregándose un caso de la segunda etapa que se desprende las dos noticias expuestas en los ejemplos primeros.

Ejemplo análisis primera etapa metodológica, noticia del periódico El Mercurio_(07-11-2010):

Documento clave para Chile ante demanda marítima de Perú en La Haya: Ecuador anunció envío de
su Carta Náutica a la ONU,
aunque sin precisar fecha

Presidente Piñera dijo que esperaba que ese documento sea entregado al organismo "lo antes posible".

RIENZI FRANCO

Enviado especial

PUERTO NATALES.- La distendida visita del Presidente de Ecuador, Rafael Correa, a la Región de Magallanes junto al Presidente Sebastián Piñera tuvo ayer un anuncio que era esperado por Chile.

Correa informó que Ecuador inscribirá su Carta Náutica ante la ONU. Este documento, publicado en agosto, grafica los límites marítimos con Perú, tomando como base los tratados de 1952 y 1954 , los mismos que Lima desconoce en su demanda contra Chile ante La Haya.

Perú presentará su réplica a la contramemoria de Santiago en dos días más.

Sin embargo, Correa no adelantó la fecha en que se realizará la inscripción: "Pero por qué tiene que haber fecha para esas cosas", comentó.

"Nosotros somos muy delicados con nuestros países hermanos, sobre todo vecinos. Entonces esperamos las observaciones de Perú. Tratamos de

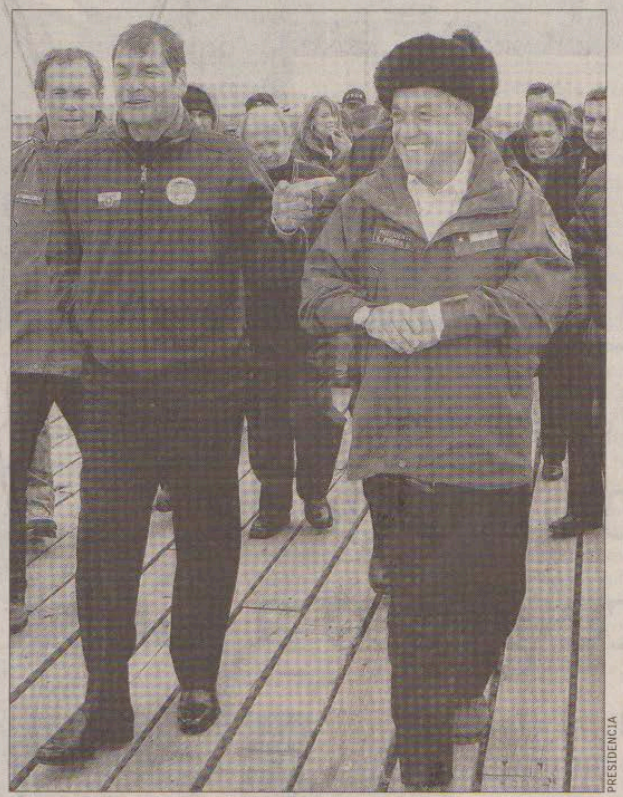

Los presidentes Correa y Piñera en Puerto Natales, previo a su frustrado viaje a la Antártica. Piñera lucía el mismo gorro que usó González Videla.

arreglar siempre las cosas por el diálogo, ajustar cualquier desinformación, cualquier desfase que tal vez haya, y luego el procedimiento normal es reeditar esa carta en la ONU", agregó Correa.

Al comentarle al canciller dría imponer a usted cuándo pedir esas pruebas de amor; usted lo decidirá por su cuenta. Nosotros, como país soberano, lo decidimos en su momento". El Presidente Pifiera, en tanto, afirmó ayer sobre la Carta Náutica que "ese documento, ese decreto y ese mapa marítimo son de público conocimiento, y esperamos que también sean entregados a Naciones Unidas lo antes posible".

La visita de Correa ha estado plagada de gestos amistosos. Ambos mandatarios estuvieron juntos casi las 48 horas, a excepción del trayecto entre Puerto Natales y Punta Arenas, donde Correa se fue en su avión privado para una sesión de su tratamiento en una rodilla.

Piñera hizo todo lo posible para llevar a Correa a la Antártica. Aunque el avión que los llevaba llegó a sobrevolar el continente blanco ayer, el mal tiempo les impidió aterrizar.

Durante la jornada, los mandatarios habían desayunado juntos en el Hotel Dreams y navegado en Puerto Natales a bordo del catamarán Horca.

Uno de los momentos más distendidos se vivió la noche del viernes, cuando ambas comitivas cenaron en el restaurante "Sotito". Se cantó "Yo vendo unos ojos negros" y el canciller Patiño hasta llevó dos guitarras para amenizar la celebración.

También hubo espacio para una breve conversación privada entre ambos presidentes. 
Comunicación intercultural mediada

\section{CUADRO 5. EJEMPLO ANÁLISIS PRIMERA ETAPA METODOLÓGICA, NOTICIA DEL DIARIO EL MERCURIO:}

\begin{tabular}{|c|c|c|}
\hline \multicolumn{2}{|c|}{ Periódico: El Mercurio / Chile } & Día: 07/11/10 \\
\hline \multicolumn{2}{|l|}{ Sección: Política } & Página: C4 \\
\hline \multicolumn{3}{|c|}{$\begin{array}{l}\text { Titular: Documento clave para Chile ante demanda marítima de Perú en La Haya: } \\
\text { ECUADOR ANUNCIÓ ENVÍO DE SU CARTA NÁUTICA A LA ONU, AUNQUE SIN PRECISAR FECHA } \\
\text {-Presidente Piñera dijo que esperaba que ese documento sea entregado al organismo “lo antes posible”. }\end{array}$} \\
\hline \multicolumn{3}{|c|}{ 1. Plano Significado/texto. Nivel temático } \\
\hline $\begin{array}{l}1.1 \text { Nivel de } \\
\text { Significados } \\
\text { Globales. }\end{array}$ & \multicolumn{2}{|c|}{$\begin{array}{l}\text { La noticia trata de la futura inscripción de la Carta Náutica de Ecuador ante la ONU. } \\
\text { Anuncio hecho por el Presidente Rafael Correa en la visita que realizó al sur de Chile } \\
\text { junto al Presidente Sebastián Piñera. El documento graficaría los límites marítimos con } \\
\text { Perú, basados en los Tratados de } 1952 \text { y 1954. Los mismos documentos que componen } \\
\text { la argumentación chilena ante La Haya. }\end{array}$} \\
\hline \multirow{4}{*}{$\begin{array}{l}1.2 \text { Nivel de } \\
\text { Significados } \\
\text { Locales. }\end{array}$} & \multicolumn{2}{|c|}{ 1.2.1 De carácter implícito o indirecto: } \\
\hline & \multicolumn{2}{|c|}{$\begin{array}{l}\text { Esta construcción noticiosa se esfuerza por dar a entender el buen ánimo entre Chile y Ecuador } \\
\text { con motivo de la presentación de la Carta Náutica de este último a la ONU, calificando además el } \\
\text { hecho y el encuentro de los mandatarios como una "celebración". } \\
\text { Incluso se utiliza el recurso de la hipérbole para exagerar el éxito de la visita de Rafael } \\
\text { Correa y las sustanciales bonanzas en las relaciones entre Chile y Ecuador: } \\
\text {-"La visita de Correa ha estado PLAGADA de gestos amistosos". } \\
\text { También hay una serie de construcciones que apuntan en la misma dirección: } \\
\text {-"la distendida visita del presidente de Ecuador"; "ambos mandatarios estuvieron juntos } \\
\text { casi las } 48 \text { horas"; "los mandatarios habían desayunado juntos"; "se cantó 'Yo vendo } \\
\text { unos ojos negros' y el Canciller Patiño HASTA llevó dos guitarras para amenizar la } \\
\text { CELEBRACIÓN". } \\
\text { Por otro lado, se plantea implícitamente que Perú no recuerda algo que es real, y que } \\
\text { tiene vigencia, puesto que los Tratados de } 1952 \text { y } 1954 \text { son los que conforman su actual } \\
\text { Carta Náutica con Ecuador. "LOS MISMOS que Lima DESCONOCE en su demanda } \\
\text { marítima contra Chile". }\end{array}$} \\
\hline & \multicolumn{2}{|c|}{ 1.2.2 De carácter explícito o directo: } \\
\hline & \multicolumn{2}{|c|}{ No se encontró. } \\
\hline \multicolumn{3}{|c|}{ 2. Plano Formal/texto-contexto. } \\
\hline $\begin{array}{l}2.1 \text { Estructuras } \\
\text { Formales } \\
\text { Sutiles. }\end{array}$ & \multicolumn{2}{|c|}{$\begin{array}{l}\text { Al calificar el viaje del Presidente Correa y su anuncio como una "celebración”, se deja } \\
\text { en la memoria del lector que Chile contaría ya con un apoyo esencial para ganar la } \\
\text { demanda marítima que le interpuso Perú ante La Haya. }\end{array}$} \\
\hline $\begin{array}{l}2.2 \text { Nivel } \\
\text { Contextual. }\end{array}$ & \multicolumn{2}{|c|}{$\begin{array}{l}\text { El marco contextual está referido a la argumentación chilena por la demanda marítima } \\
\text { que le interpuso Perú ante La Haya, que descansa en los tratados de } 1952 \text { y 1954, sobre } \\
\text { los que también regiría la Carta Náutica de Perú y Ecuador. } \\
\text {-El tratado de } 1952 \text { fue suscrito en Santiago de Chile por Ecuador, Perú y Chile y } \\
\text { denominado "Declaración de Zona Marítima". En el documento se establece el } \\
\text { reconocimiento de las } 200 \text { millas náuticas de cada país, cuya definición corresponde a un } \\
\text { minuto de arco sobre el paralelo de la línea del Ecuador. Mientras que en 1954, estos } \\
\text { mismo países deciden firmar un nuevo documento llamado "Convenio sobre Zona } \\
\text { Especial Marítima", estableciéndose una zona especial a partir de las } 12 \text { millas marinas } \\
\text { de la costa, de } 10 \text { millas marinas de ancho a cada lado del paralelo que constituye el } \\
\text { límite marítimo. }\end{array}$} \\
\hline
\end{tabular}


Ejemplo análisis primera etapa metodológica, noticia del periódico El Comercio (07-11-2010):

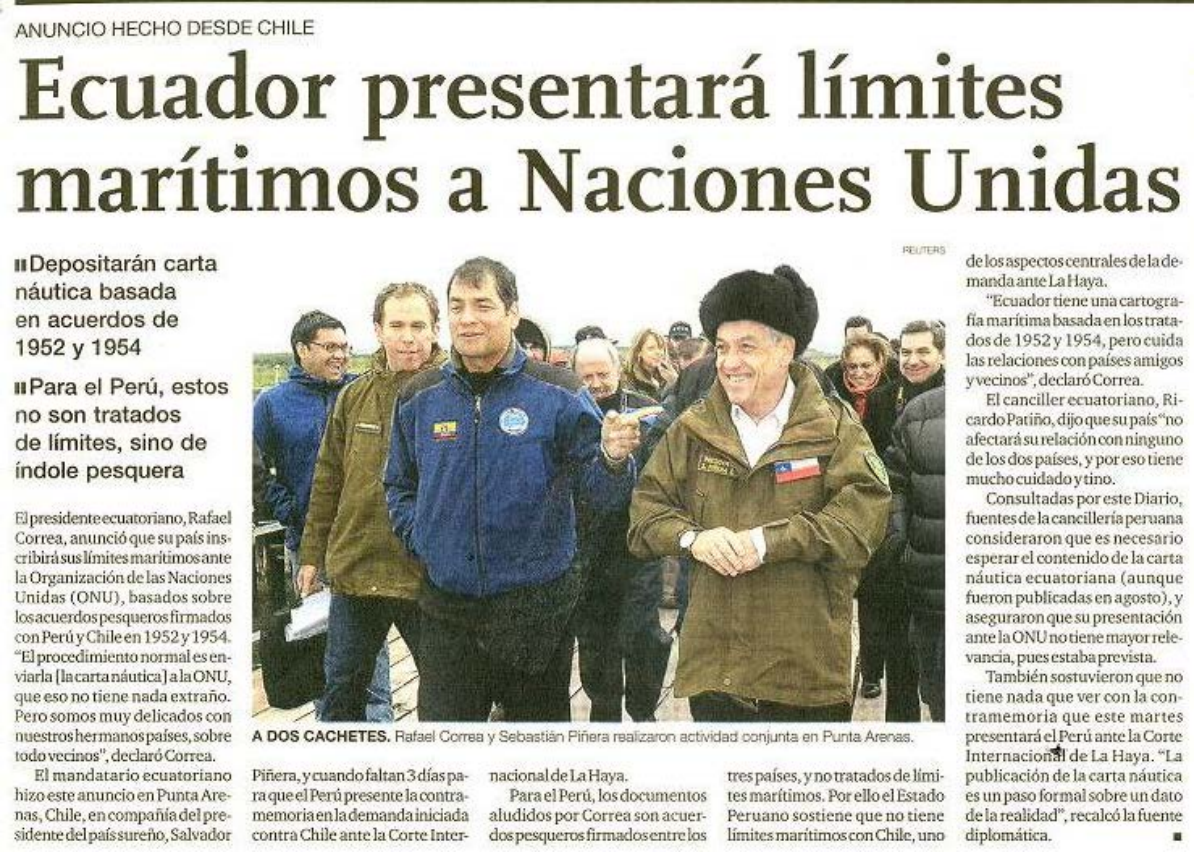




\section{CUADRO 6. EJEMPLO ANÁLISIS PRIMERA ETAPA METODOLÓGICA, NOTICIA DEL PERIÓDICO EL COMERCIO:}

\begin{tabular}{|c|c|c|}
\hline \multicolumn{2}{|c|}{ Periódico: El Comercio / Perú } & Día: 07/11/10 \\
\hline \multicolumn{2}{|c|}{ Sección: Política } & Página: a6 \\
\hline \multicolumn{3}{|c|}{$\begin{array}{l}\text { Titular: Anuncio hecho desde Chile } \\
\text { ECUADOR PRESENTARÁ LÍMITES MARÍTIMOS A NACIONES UNIDAS } \\
\text {-Depositarán Carta Náutica basada en acuerdos de } 1952 \text { y } 1954 . \\
\text {-Para el Perú estos no son tratados de límites, sino de índole pesquera. }\end{array}$} \\
\hline \multicolumn{3}{|c|}{ 1. Plano Significado/texto. Nivel temático } \\
\hline $\begin{array}{l}\text { Nivel de } \\
\text { Significados } \\
\text { Globales. }\end{array}$ & \multicolumn{2}{|c|}{$\begin{array}{l}\text { En la voz del medio, se entrega información sobre la Carta Náutica que presentará Ecuador } \\
\text { ante la ONU vinculándola con la demanda marítima interpuesta por Perú sobre Chile. Se } \\
\text { especifican los límites marítimos de Ecuador basados en los acuerdos de } 1952 \text { y 1954, que - } \\
\text { según se indica- "para el Perú no son tratados de límites, sino de índole pesquera". } \\
\text { Mientras por otro lado, fuentes de la cancillería peruana imparten seguridad señalando que } \\
\text { la carta de límites ecuatorianos no tiene relevancia en la contramemoria del Perú porque } \\
\text { estaba prevista. }\end{array}$} \\
\hline \multirow{4}{*}{$\begin{array}{l}\text { Nivel de } \\
\text { Significados } \\
\text { Locales. }\end{array}$} & \multicolumn{2}{|c|}{ De carácter implícito o indirecto: } \\
\hline & \multicolumn{2}{|c|}{$\begin{array}{l}\text { Se utiliza la expresión "A dos cachetes” para señalar de forma implícita que Rafael } \\
\text { Correa estaría actuando a dos bandos, por un lado con Perú y por otro con Chile. Se } \\
\text { desprende también que Ecuador ya no es un país confiable. } \\
\text {-”A DOS CACHETES. Rafael Correa y Sebastián Piñera realizaron actividad } \\
\text { conjunta en Punta Arenas" (pie de fotografía). } \\
\text { Implícitamente se da a entender que el Presidente de Ecuador apoya a Chile, por } \\
\text { anunciar su Carta Náutica en Chile y a sólo tres días de que Perú entregue su réplica } \\
\text { ante La Haya. También se alude al Presidente Sebastián Piñera, con lo que parece ser } \\
\text { un error tipográfico: "Salvador Piñera". } \\
\text {-”El Mandatario ecuatoriano HIZO ESTE ANUNCIO en Punta Arenas, Chile, en } \\
\text { compañía del Presidente del país sureño, Salvador Piñera, Y CUANDO FALTAN } 3 \\
\text { DÍAS para que el Perú presente la contramemoria en la demanda iniciada contra Chile } \\
\text { ante La Corte Internacional de La Haya”. } \\
\text { Se minimiza el anuncio de Ecuador, dando a entender que Perú ya lo había previsto, por lo } \\
\text { que posee control en relación a la demanda marítima interpuesta a Chile. } \\
\text {-”Fuentes de la cancillería peruana... aseguraron que su presentación ante la ONU } \\
\text { no tiene mayor relevancia, pues estaba prevista”. }\end{array}$} \\
\hline & \multicolumn{2}{|c|}{ De carácter explícito o directo: } \\
\hline & \multicolumn{2}{|c|}{$\begin{array}{l}\text { De forma directa se desecha la postura chilena: } \\
\text {-"Para el Perú, los documentos aludidos por Correa SON ACUERDOS pesqueros } \\
\text { firmados entre los tres países, y NO TRATADOS DE LÍMITES MARÍTIMOS”. } \\
\text {-"EL ESTADO PERUANO SOSTIENE QUE NO TIENE LÍMITES MARÍTIMOS CON } \\
\text { CHILE, uno de los aspectos centrales de la demanda ante La Haya". }\end{array}$} \\
\hline \multicolumn{3}{|c|}{ 2. Plano Formal/texto-contexto. } \\
\hline $\begin{array}{l}\text { Estructuras } \\
\text { Formales } \\
\text { Sutiles. }\end{array}$ & \multicolumn{2}{|c|}{ No se encontró. } \\
\hline $\begin{array}{l}\text { Nivel } \\
\text { Contextual. }\end{array}$ & \multicolumn{2}{|c|}{$\begin{array}{l}\text { El marco contextual remite a la desconfianza, al doble juego de Ecuador por presentar su Carta } \\
\text { Náutica ante la ONU, considerando que en los últimos meses la buena relación entre Perú y } \\
\text { Ecuador era algo destacado en este mismo medio de comunicación. La Carta Náutica se basa } \\
\text { en los tratados suscritos por Perú, Chile y Ecuador en } 1952 \text { y 1954. Tratados que componen } \\
\text { parte central de la argumentación chilena en el diferendo marítimo entre Perú y Chile, } \\
\text { evidenciándose, de este modo, el apoyo a Chile por parte de Ecuador. }\end{array}$} \\
\hline
\end{tabular}


CUADRO 7. EJEMPLO ANÁLISIS SEGUNDA ETAPA METODOLÓGICA, DESPRENDIDO DE LOS EJEMPLOS DE ANÁLISIS DEL PRIMER PASO METODOLÓGICO:

\begin{tabular}{|c|c|c|c|c|c|c|c|}
\hline \multicolumn{4}{|c|}{ CHILE } & \multicolumn{4}{|c|}{ PERÚ } \\
\hline \multicolumn{4}{|c|}{$\begin{array}{l}\text { Título: Documento clave para Chile ante demanda } \\
\text { marítima de Perú en La Haya: } \\
\text { Ecuador anunció envío de su Carta Náutica a la } \\
\text { ONU, aunque sin precisar fecha }\end{array}$} & \multicolumn{4}{|c|}{$\begin{array}{l}\text { Título: Anuncio hecho desde Chile: } \\
\text { Ecuador presentará límites marítimos a Naciones } \\
\text { Unidas }\end{array}$} \\
\hline \multicolumn{8}{|c|}{ Identificación y comportamiento de variables temáticas sobre un mismo acontecimiento } \\
\hline $\begin{array}{c}\text { Difer } \\
\text { ente }\end{array}$ & Opuesto & Complementario & Igu & & Complementario & Opuesto & $\begin{array}{l}\text { Difer } \\
\text { ente }\end{array}$ \\
\hline \multicolumn{8}{|c|}{ 1. Plano Significado/texto. Nivel temático } \\
\hline \multicolumn{8}{|c|}{ 1.1 Nivel Global } \\
\hline \multicolumn{8}{|c|}{\begin{tabular}{|c|c|} 
de Ecuador & \\
\end{tabular}} \\
\hline \multicolumn{8}{|c|}{ 1.2.1 Nivel Local Implícito } \\
\hline & $\begin{array}{l}\text { Legalidad } \\
\text { argumen- } \\
\text { tación } \\
\text { chilena }\end{array}$ & & \multicolumn{2}{|c|}{$\begin{array}{c}\text { Apoyo } \\
\text { internacio-nal } \\
\text { de Ecuador a } \\
\text { Chile }\end{array}$} & $\begin{array}{l}\text { Desconfianza de } \\
\text { Perú hacia } \\
\text { Ecuador / Control } \\
\text { peruano de la } \\
\text { situación }\end{array}$ & $\begin{array}{l}\text { Arbitra- } \\
\text { riedad } \\
\text { argu- } \\
\text { mentación } \\
\text { chilena }\end{array}$ & \\
\hline \multicolumn{8}{|c|}{ 1.2.1Nivel Local Explícito } \\
\hline & & & & & & & \\
\hline \multicolumn{8}{|c|}{ 2. Plano Formal/texto-contexto. } \\
\hline \multicolumn{8}{|c|}{ 2.1 Estructura Formal sutil } \\
\hline & & $\begin{array}{c}\text { Posible fallo } \\
\text { favorable a Chile }\end{array}$ & & & & & \\
\hline \multicolumn{8}{|c|}{ 2.2 Nivel Contexto } \\
\hline & $\begin{array}{c}\text { Legalidad } \\
\text { argumental } \\
\text { (Tratados } \\
1952 \text { y 1954) }\end{array}$ & & $\begin{array}{r}\text { Tens } \\
\text { bilate } \\
\text { Procesc } \\
\text { de lín }\end{array}$ & $\begin{array}{l}\text { ión } \\
\text { eral/ } \\
\text { o legal } \\
\text { nites }\end{array}$ & $\begin{array}{l}\text { Desconfianza de } \\
\text { Perú hacia } \\
\text { Ecuador }\end{array}$ & & \\
\hline
\end{tabular}

\section{CONCLUSIONES}

Son muchas las relaciones y reflexiones que se desprenden de la investigación que sustenta este trabajo, motivo por el que intentaremos expresar las ideas centrales del mismo. Partimos señalando fehacientemente que la hipótesis planteada en este estudio fue comprobada: los medios resultan ser uno de los principales constructores de representación social de realidad. Evidenciamos que se promueven estereotipos $\mathrm{y}$ prejuicios direccionados a potenciar la institucionalización de una identidad y otra alteridad en torno a los discursos de la diferencia entre Chile y Perú.

Tanto El Mercurio como El Comercio construyen realidades a partir de imaginarios aprendidos y establecidos por los discursos de autoridad, productos de tradiciones socio-histórico-culturales, que tienden a favorecer y fortalecer identidades propias por sobre las ajenas. En relación al objetivo planteado, se logró efectivamente comprender los procesos de construcciones noticiosas que los medios analizados 
hacen sobre los discursos de la diferencia, considerando para ello los estudios desarrollados desde la comunicación mediada y sus aplicaciones en el periodismo.

\subsection{EN RELACIÓN AL ACD}

Comprobamos que El Mercurio suele recurrir a situaciones sociales, políticas, económicas e históricas para acentuar la superioridad de Chile, sus gobiernos y ciudadanos, por sobre Perú. Para ello utiliza estrategias gramaticales discursivas implícitas y sutiles, en tanto, en escasas oportunidades este medio se jacta directamente de dicha superioridad en secciones que no sean de opinión. Agregamos que El Mercurio construye sus discursos sobre la polarización Nosotros/Ellos, adscribiéndose a un "nosotros los chilenos" en relación a un "ellos los peruanos".

Por su parte, El Comercio apunta principalmente a situaciones socio-políticas e históricas en favor de la unidad nacional, destacando su proceder pacífico y correcto por sobre Chile. El Comercio también acude a la polarización Nosotros/Ellos como una constante, llegando a identificarse explícitamente como medio nacionalista al referirse al diferendo marítimo que sostienen Chile y Perú: "El Comercio, fiel a su tradición de invariable defensor de los derechos de nuestro país, de aquellos asuntos que constituyen una política de Estado, un objetivo nacional, del cual nadie puede sentirse distante ni ajeno...” (Publicación El Comercio, 24-11-2010:a4).

Los medios analizados fortalecen las respectivas identidades de sus EstadoNación, a través de la construcción de acontecimientos noticiosos concernientes a Chile y Perú, promoviendo modelos mentales que apuntan hacia los hechos más negativos comunes o relacionales a ambos países.

\subsection{ACERCA DEL ACCD}

Mediante la herramienta de análisis crítico comparativo, apreciamos que en los procesos recíprocos de construcción social de realidad, ambos medios abordan una serie de variables temáticas comunes que articulan sus discursos pero que se construyen en confrontación entre Chile y Perú, remitiendo a desconfianzas mutuas, tensiones bilaterales y apelando a sus respectivos nacionalismos y contextos históricos. Estas tensiones se despliegan principalmente en torno al actual proceso legal de límites marítimos que sostienen ambos países ante La Haya, interviniendo discursivamente una serie de variables opuestas como: legalidad-arbitrariedad, superioridad-inferioridad y también variables de carácter complementarias como: ambición-desconfianza y conflictividad-tensión bilateral, entre otras.

Pese a que ciertas narraciones discursivas se generan sobre la base de los mismos hechos noticiosos, existe un esfuerzo por enfatizar una visión deficitaria de la relación binacional. Del análisis comparativo se evidencia, que El Mercurio trae a sus discursos variables negativas de carácter diferentes o ajenas al tema central publicado en ambos medios, como: inseguridad, desempleo, inmigración, nivel socioeconómico, conflictividad y populismo, apuntando todas a construcciones 
negativas en relación al Perú, sus gobiernos y ciudadanos. En tanto El Comercio, manifestando igualmente un conjunto de variables negativas que apuntan hacia Chile, se ajusta más a variables opuestas o complementarias a la temática central equivalente a la publicación de ambos medios.

"Los estudios comparativos de las noticias internacionales sugieren que la 'misma' historia tiende a ser presentada de manera diferente a distintas sociedades, pues son adaptadas a contextos sociales particulares” (Israel, 2006:77). En el caso de Chile y Perú tal parece que sus contextos sociales particulares son íntimamente complementarios entre sí. En el transcurso del análisis confirmamos que ambos medios auxilian sus discursos en el marco contextual periódico de la vieja Guerra del Pacífico. Como esbozamos con antelación, este conflicto bélico ha tenido gran injerencia en las conformaciones identitarias nacionales de y entre ambos países, evidenciándose notoriamente las mutuas desconfianzas históricas.

\subsection{REFLEXIONES FINALES Y PROPUESTAS NO CONCLUYENTES}

Van Dijk señala que el contexto no es una situación objetiva y externa al discurso, sino más bien una construcción, una definición subjetiva de los participantes y de las dimensiones relevantes de la situación comunicativa (2009:167). Los discursos informativos influyen sobre los modelos mentales estableciendo un consenso dominante e instaurando un control hegemónico respaldado por otros discursos de élite. En este caso hablamos de control ilegítimo no sólo del discurso, sino también del contexto.

Como un muerto tibio que nunca se enfría, así se presenta la memoria histórica oficial de un registro bélico con serias distorsiones en Chile y Perú; promovido desde los Estados y transmitido por los diversos canales oficiales en el espacio de la reproducción social y organización de la diferencia, configurando un orden institucional a la vez que un modelo contextual. Este orden es mantenido y reforzado por los medios, que se presentan como una de las principales instituciones de reproducción ideológica. De acuerdo con van Dijk (1997), creemos incluso que son más decidores que los sistemas educativos, pero los medios y los comunicadores no se hacen a dicha tarea en solitario. Ante estas relaciones, y de acuerdo a los resultados de la investigación, nos parece válido y oportuno preguntar y visibilizar ¿Qué motiva el mantenimiento y reproducción de la conflictividad entre Chile y Perú?

Esta conflictividad beneficia, desde luego, a los militares de ambos países que así pueden engordar sus presupuestos; a los que les venden armas y pertrechos; y a los políticos, que pueden beneficiarse de un tema que es sensible a la población. "Fuera los chilenos" o "fuera los peruanos" son lemas que conquistan votos en ambos lados de la línea de la concordia (Carlos Contreras. Entrevista personal, 2010). 
Tomando la cita anterior y con el objeto de ejemplificar las reflexiones recurrentes frente a lo planteado, se presenta aquí una voz desde Chile y otra del Perú. Como se puede apreciar poco importa la nacionalidad de las ideas expresadas, ya que parecen narrar una misma escena.

Esto beneficia a los sectores militares, la existencia de conflictos reales o ficticios justifica gastos en armas que supera con creces lo que estos países debiesen tener y genera esta especie de mito de tener estas poderosísimas Fuerzas Armadas, que extrañamente ambas en el siglo XX le disparan a connacionales. ¿Cómo puede ser que franceses y alemanes que tenían grandes conflictos, sí puedan generar sistemas de pacificación y resguardo civil? No cabe duda que detrás de esto están oscuros intereses militares y comerciales, no tenemos una prensa tan libre, las cosas las podemos decir sólo en las tesis (Fernando Ramírez. Entrevista personal, 2010).

A través de una multiplicidad de relaciones que se desprenden de esta investigación, señalamos que los medios estudiados no parecen sólo empresas autorreferenciales que defienden intereses vinculados exclusivamente a la comunicación. Haremos notar que tanto El Mercurio de Chile como El Comercio de Perú integran la Sociedad Interamericana de Prensa (SIP) que reúne a los grandes negocios mediáticos que defienden intereses concentrados corporativos y que, según el filósofo argentino Ricardo Forster, “están profundamente entramados en los valores ideológicos políticos de las derechas continentales, representan una visión del mundo, una visión de sociedad, por lo que hay que desconfiar y pensar en lo que nos están diciendo" (El Racismo de la SIP, programa 6,7,8 de Canal 7 TV Publica de Argentina).

Precisamos que, ante la lamentable concentración de los medios de alta lectoría en grandes grupos económicos y políticos, se obliga a una parte importante de lectores a interpretar en base a intereses fijados por medio de códigos instaurados por quienes ostentan el poder. Sabemos que combatir la promoción de escenarios etnocéntricos y nacionalistas tendientes a fomentar modos de intolerancia y supremacías socioculturales, es labor de hormigas frente a manos negras, poderosas, gigantes y decididas a blandir el puño.

Para Israel Garzón, “en el marco de las relaciones internacionales y de una pedagogía activa, que reivindica la diferencia dentro de la igualdad de derechos y oportunidades se encuentra la educación en comunicación intercultural” (2006:72). Por ello, abogamos por esta propuesta como una alternativa viable para cambiar actuales construcciones sesgadas, en lo que Rodrigo Alsina denomina mundos posibles y, con esto, influir de forma integracionista en los potenciales mundos de referencia (1989:188-190).

Y como toda labor de hormigas, se vuelve débil e infructuosa en solitario, debemos propiciar una educación en comunicación intercultural en conjunto con otras áreas de estudio, aunar esfuerzos contra de los diferentes discursos de 
dominación social y no únicamente, sentarnos a pensar y debatir la comunicación intercultural entre institutos, escuelas, salones y personas abocadas a la comunicación. La comunicación intercultural debe abrirse a nuevos espacios.

Por último y como parte fundamental de este mismo estudio, abogamos también en favor de los análisis críticos de los diferentes discursos, como herramientas efectivas de resistencia y cambio social:

Un análisis crítico de calidad no solamente contiene principios, sino que también es práctico, efectivo, y por lo tanto, flexible; no solamente se dirige a la comprensión teórica, sino que además aporta sugerencias, soluciones y alternativas, y se aplica por lo tanto en la formulación de antiideologías y estimula la resistencia. En efecto, no solamente quiere describir el mundo, sino que desea cambiarlo (van Dijk, 1997:51).

Dado que la comunicación humana es un proceso de artificialidad —en el sentido de que es una construcción del hombre - creemos que es absolutamente posible romper con el orden institucional en expansión y con la dominación que de allí se esgrime, direccionando actuales construcciones de la realidad desde la diferencia y en favor del quebrantamiento de tradiciones institucionales y por completo etnocéntricas.

Universidad Austral de Chile* Facultad de Filosofía y Humanidades. Escuela de Graduados Isla Teja S/N, CP 5090000, Valdivia (Chile) rodrigobrowne@uach.cl

Universidad Católica del Norte** Facultad de Humanidades. Escuela de Periodismo Av. Angamos 0610, CP 1270709, Antofagasta (Chile) cyanezduamante@ucn.cl 
Comunicación intercultural mediada

\section{BIBLIOGRAFÍA}

Acosta, Ángel. La Bisagra. Ensayo sobre complejidad, crisis y comunicación. Sevilla: Alfar, 2010.

Berger, Peter L.; Luckmann, Thomas. La Construcción Social de la Realidad. Buenos Aires: Amorrortu, 1972.

Bourdieu, Pierre. Sociología y Cultura. México: Grijalbo, 1990.

Browne, Rodrigo; Del Valle, Carlos; Silva, Víctor; Carvajal, Julio; e Inzunza, Alex. "Propuesta teórico-metodológica para un análisis crítico y complejo del discurso (ACCD) en la prensa de Chile y Perú. El ejemplo de 'La Cuarta' y 'Ajá'”. Estudios sobre el Mensaje Periodístico, ํㅜ 12, pp. 89-101. Madrid, 2011.

Browne, Rodrigo; Silva, Víctor; Baessolo, Ricardo. "Periodismo intercultural: representación peruana y boliviana en la prensa chilena”. Comunicar, $\mathrm{N}^{\circ} 36$, Huelva, 2010:33-41.

Browne, Rodrigo. "Comunicación Intercultural y Periodismo de Actualidad. (In)disciplinas e (in)definiciones para una deconstrucción crítica de los medios”. Comunicación, № 4, Sevilla, 2006:223-242.

Contreras, Carlos. "Memoria y verdad sobre la guerra con Chile. Una reflexión acerca de las posibilidades de cambiar la historia”. Revista Coyuntura. Análisis Económico y Social de Actualidad, № 8. Perú, 2006:10-11.

$<$ http://cisepa.pucp.edu.pe/coyuntura/08.pdf>, visitado el 10 de septiembre de 2010.

Contreras, Carlos; Cueto, Marcos. Historia del Perú Contemporáneo, Instituto de Estudios Peruanos (4ta Edición): Lima, 2007.

Contreras, Carlos. Entrevista personal, 20 de diciembre de 2010.

Cortéz, Guillermo. Entrevista personal, 24 de noviembre de 2010.

Dager, Joseph en Somarriva, Marcelo. "Enemigos íntimos". El Mercurio Online, Sección Artes y Letras, 17 de abril de 2005.

$<$ http://www.n-cl.ch/noticias.htm>, Consultado el 23 de agosto de 2010.

Del Valle, Carlos; Miranda, Eduardo; Ortiz, Manuel; Agüero Claudio; y Guínez, Rubén. "Comunicación, discurso y derecho: análisis comparado, mediante software, del discurso de sentencias penales y de noticias policiales (Temuco, Chile)". Comunicación informativa y nuevas tecnologías, Gran Aldea. Buenos Aires, 2009:139-151.

Del Valle, Carlos; Mayorga, Alberto; y Nitrihual, Luis. "Prensa, justicia y producción narrativa del poder: fundamentos teórico-metodológicos para un estudio comparado del discurso”. Convergencia, vol.17, № 54. México 2010:195-198.

Flusser, Vilém. "Qué es comunicación”. Kommunikologie, Main: Frankfurt, 2003. Traducción de Breno Onetto Muñoz.

Forster, Ricardo. Informe “El Racismo de la SIP”, programa 6, 7, 8 de Canal 7 TV Publica de Argentina. Entrevista de archivo emitida el 24 de noviembre de 2010.

García-Canclini, Néstor. Diferentes, Desiguales y Desconectados. Mapas de la Interculturalidad. Gedisa: Barcelona, 2004.

Geertz, Clifford. La Interpretación de las Culturas. Gedisa: Barcelona, 1973.

Israel Garzón, Estrella. Comunic@ción y Periodismo en una Sociedad Global. Comunicar la diferencia. Trillas: México, 2006. 


\section{Rodrigo Browne y Constanza Yáñez}

Mayorga, Alberto y León, Carla. "El malvado siempre es el otro: Perú y su construcción discursiva en la prensa chilena”. Universum, $\mathrm{N}^{\circ} 22$ vol.2. Universidad de Talca, 2007:170-182.

Rodrigo, Miquel. Teorías de la Comunicación: Ámbitos, métodos y perspectivas. Aldea Global: Barcelona, 2001.

Comunicación Intercultural. Anthopos: Barcelona, 1999.

La Construcción de la Noticia. Paidós: Barcelona, 1989.

Ramírez, Fernando y Silva, Victoria. Historia, Geografía y Ciencias Sociales. Texto para el estudiante. MN Editorial, Ministerio de Educación: Chile, 2009.

Ramírez, Fernando. Entrevista personal, 22 de diciembre de 2010.

Silva, Víctor y Browne, Rodrigo. Antropofagias. Las indisciplinas de la comunicación. Biblioteca Nueva: Madrid, 2007.

Touraine, Alain ¿Podremos vivir juntos? Iguales y diferentes. PPC: Madrid, 1997.

Van Dijk, Teun. Discurso y Poder. Gedisa: Barcelona, 2009.

------ Racismo y análisis crítico de los medios. Paidós: Barcelona, 1997.

Yáñez Constanza. (2010, noviembre y diciembre). Entrevista con Fernando Ramírez, historiador y docente de la Universidad de Chile; con Carlos Contreras, profesor de historia económica de la Pontificia Universidad Católica de Perú; y con Guillermo Cortez, Dr. En Historia y Director del Museo Regional de Atacama. Comunicación intercultural mediada de carácter binacional. Construcción de la noticia a través de un análisis crítico y complejo del discurso en la prensa diaria de cobertura nacional de Chile y Perú. Tesis de Maestría no publicada, Universidad Austral de Chile, 2011. 